\title{
Advanced Human Behavioral Cues Detection: A Method in Social Signal Processing
}

\author{
K.Sunilkumar ${ }^{1}$ and Dr.T.KishoreKumar ${ }^{2}$ \\ ${ }^{1}$ Department of Electronics and Communication Engineering, VITS, \\ Hasanparthy,Warangal,A.P, INDIA \\ sunil_veena10@yahoo.co.in \\ ${ }^{2}$ Department of Electronics and Communication Engineering,NITWarangal,A.P, INDIA \\ kishorefregmail.com
}

\begin{abstract}
Social Signal Processing (SSP) is the new research domain that aims at understanding social interactions and social intelligence. The essence of social intelligence is the ability to recognize human social signals and behaviors cues (verbal and non-verbal). We present a unified model for non-verbal behavioral cues SSP under the categories of face detection like eyes-mouth-lips-ears-nose, emotional assessments like anger-happy-sadness-neutral and physical appearance like height (short-average-tall), attractiveness (smartness) and body shape(slim-normal-flat) in real-world cluttered images which is one of the method in social signal processing. It is rather difficult task to detect and interpret temporal patterns of nonverbal behavioral cues in a given context. Our main objective is to detect non-verbal behavioral cues by introducing advanced human behavioral cues detection method. Our proposed method shown a better results and practically applicable in the real world environment. We implemented our proposed method in MATLAB R2007b simulator and designed a GUI for our proposed method.
\end{abstract}

\section{KEYWORDS}

SSP, social interactions, social intelligence, behavioral cues, cluttered images, MAT LAB.

\section{INTRODUCTION}

A social signal is a signal that provides information about "social facts", i.e., about social interactions, social emotions, social attitudes, or social relations $[2,4]$. Social interaction is a specific event in which an agent A performs some social actions directed at another agent that is actually or virtually present. Social interactions may be mediated by communicative and informative signals.

Social emotions: A clear distinction can be made between individual and social emotions. The latter can be defined as an emotion that an Agent A feels toward andAgent B. Happiness and sadness are typical examples of individual emotions - we can be happy or sad on our own; our feelings are not directed to any other person. On the other hand, admiration, envy, and compassion are typical examples of social emotions - we have these feelings toward another person. Signals revealing individual emotions of a person and those communicating social emotions both include facial expressions, vocal intonations and outbursts, body gestures and postures, etc.

Social evaluation: Social evaluation of a person relates to assessing whether and how much the characteristics of this person comply with our standards of beauty, intelligence, strength, justice, altruism, etc.

Social attitudes: The notion of attitude has been widely investigated in Social Psychology. Social attitude can be defined as the tendency of a person to behave in a certain way toward

DOI : 10.5121/ijscai.2013.2103 
another person or a group of people. Social attitudes include cognitive elements like beliefs, evaluations, opinions, and social emotions.

Social relations: A social relation is a relation between two (or more) persons in which these persons have common or related goals, that is, in which the pursuit, achievement, or thwarting of a goal of one of these persons determines or is determined in some way by the pursuit, achievement, or thwarting of a goal of the other involved person.

Social signal processing (SSP) is the new research and technological domain that aims at providing computers with the ability to sense and understand human social signals and behavioral cues (verbal and non-verbal). Non-verbal behavior is a continuous source of signals which convey information about feelings, mental state, personality, and other traits of people.

Our paper begins with classifying different types of non-verbal behavioral cues. And detecting social non-verbal behavioral cues like face detection, emotional assessments and physical appearance. The rest of the papers as follow in chapter 2 the non-verbal human behavioral cues are explained. In chapter 3 the analysis of SSD is shown and in chapter 4 the implementation of proposed method had given and simulation results are illustrated in chapter5 results. Simulated results are discussed with the proposed method in discussion chapter 6 . Followed by conclusions and future research work.

\section{NON-VERBAL HUMAN BEHAVIORAL CUES}

Non-verbal human behavioral cues detection involves physical appearance, gesture and posture, face and eyes behavior, vocal behavior and space and environment. In our paper we are proposing a unified model to detect physical appearance, face and eyes behavior and emotional assessments [1,3].

The physical appearance includes natural characteristics such as height, body shape, physiognomy, skin and hair color, as well as artificial characteristics such as clothes, ornaments, makes up, and other manufacts used to modify/accentuate the facial/body aspects. The main social signal associated to physical appearance is the attractiveness and body shape. The human face is involved in an impressive variety of different activities. It houses the majority of our sensory apparatus: eyes, ears, mouth, and nose, allowing the bearer to see, hear, taste, and smell. Most facial-expressions analyzers developed so far target human facial affect analysis and attempt to recognize a small set of prototypic emotional facial expressions like happiness and anger. Our paper deals with detecting the social behaviors in the above behavioral cues which is shown in table 1.

Table 1: social signal detection issues

\begin{tabular}{|c|c|c|c|}
\hline Social cues & \multicolumn{3}{|c|}{ Social behaviours } \\
\hline $\begin{array}{c}\text { Physical } \\
\text { Appearance }\end{array}$ & Emotion & Personality & Status \\
\hline Height & - & - & Yes \\
\hline Attractiveness & - & Yes & Yes \\
\hline Body Shape & - & Yes & Yes \\
\hline Face and & Emotion & Personality & Status \\
\hline
\end{tabular}




\begin{tabular}{|c|c|c|c|}
\hline $\begin{array}{c}\text { eyes } \\
\text { behavior }\end{array}$ & Yes & Yes & Yes \\
\hline $\begin{array}{c}\text { Facial } \\
\text { expressions }\end{array}$ & Yes & Yes & Yes \\
\hline $\begin{array}{c}\text { Focus of } \\
\text { attention }\end{array}$ & Yes \\
\hline
\end{tabular}

\section{ANALYSIS OF SOCIAL SIGNAL DETECTION (SSD)}

In our paper we had analysed the give image into different assessments depending on the levels of extraction of the cue detections. Each detection involves separate detection steps to retrieve the desired feature. For that reason, we suggest several intermediate-level mechanisms which operate upon the base level representations. We reliably estimate the head pose in one processing step. Four different facial features are detected and localized in the images of a stereo pair. These enable the detection of the eyes and the mouth corners within the images of a stereo pair. After successful localization of intermediate level facial features, head and its orientation has been detected the search for the expected regions of the upper and lower part of the face i.e., lips, ears and nose.

The expected regions are processed in the next stage for emotional assessments, for that the skin mapping technique is used. In proposed skin mapping technique the emotional assessments like anger, happy, sadness and neutral will be detected and the percentage of emotional feelings in the face image will be detected [5].

The emotional assessment features of face are processed in the last stage of human cues detection method. Here the facial expressions will be no where related to the physical appearance. In this last stage, using the body postures and gestures detection by analysing the arm movement, head inclinations, body edges, limb motions, shifting postures the detection of physical appearances [6] like height, attractiveness and body shape will be detected.

The above discussed process methods for human cues detection can be analysed as:

- Read image (face or complete body posture) from the database.

- Detect the face features by mapping the Eyes, Mouth, Lips, Ears and Nose using localization process.

- In localization process the facial expressions are analyzed based on the triangular and rectangular illustration of the face features. These features will be detected as face emotional characters for the next stage of cues detection.

- Input the detected face image to extract the emotional assessments of the face.

- Detect the emotional assessments like Anger, Happy, Sadness and Neutral using skin mapping technique.

- In skin mapping technique, the skin color segmentation will be detected based on the intensity of emotional assessments of the face.

- Input the detected emotional assessed image to extract the physical appearance of the given body posture.

- Detect the physical appearance by body postures and detection method.

- The body postures detection will be done depending on the curves and space environment of the human body. The detection will be estimated based on the side and diagonal human body postures image. 
- The physical appearance will be detected based on the face edge detection, trapezoidal point detection and counter localization of the emotional detected face image.

- The result image will be having complete proposed human cues face, emotional and physical detection.

- The three stage detection provides a reliable and accurate detection methodology.

\section{IMPLEMENTATION OF PROPOSED METHOD FOR SOCIAL SIGNAL DETECTION}

The detection of social signal for human behavior cues are implemented based on the techniques and process discussed in the analysis of social signal processing. The implementation of proposed method is clearly explained by the block diagram of SSD.The proposed method of human cues detections can be clearly shown in fig 4.1 and 4.2.

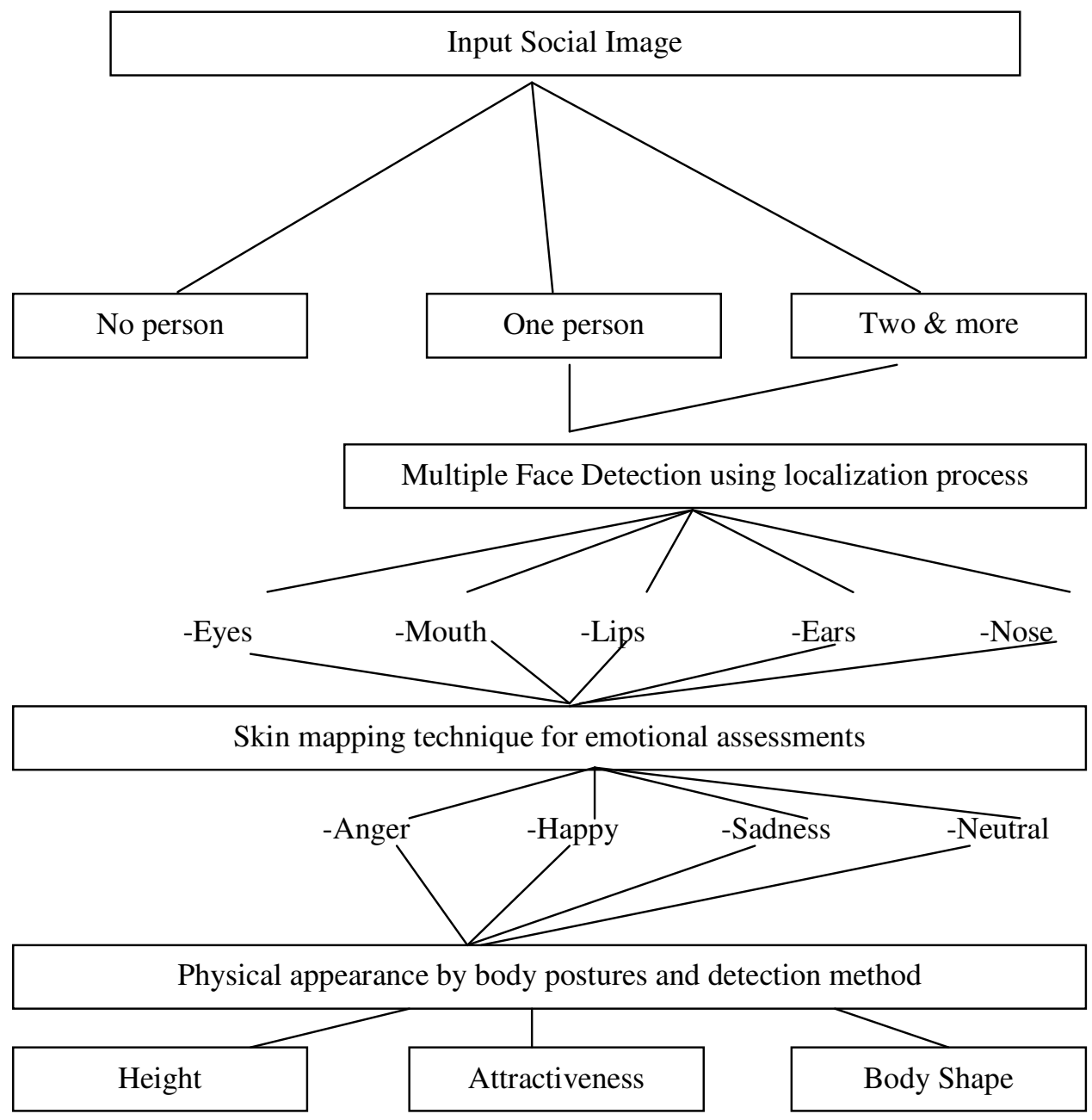

Figure 4.1: The proposed methods of human cues detections. 


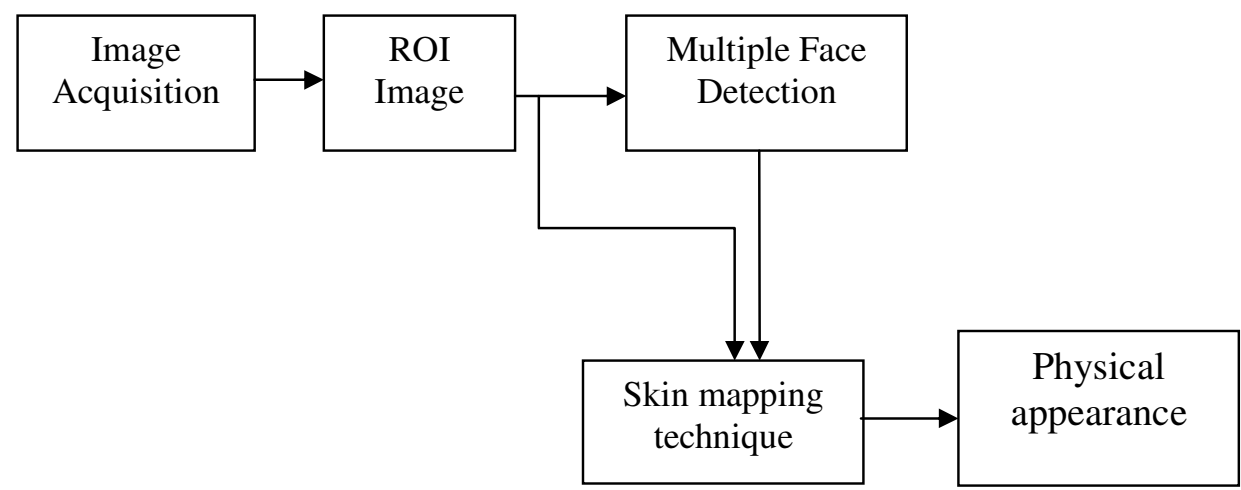

Figure 4.2: Block Diagram of our proposed method.

In our method, input image of different facial expressions will be taken. First the detection of face features will be done. These features will be given to the second stage of proposed method to extract the emotional assessments of the face feature extracted image. Next the physical appearance will be extracted. We implemented our proposed method in MATLAB R2007b GUI feature Simulink. Using MATLAB GUI simulink the input test data of 50 are taken for analysis. The extracted features are analyzed for feature point's extraction. These feature points will extract the appearance and posture information of the given image.

\section{RESULTS}

Using MATLAB GUI simulink we have implemented our proposed method of social signal processing. In GUI we have taken 50 images to implement our method. In these 50 images, 15 images are single face image with different facial expressions and 35 images are multiple face images with different emotional and physical appearances. Our proposed method has an advantage to take all types of images to find the desired feature of the given image. Images which we have taken for our analysis are tabulated below.

Table 5.1: Input face emotions

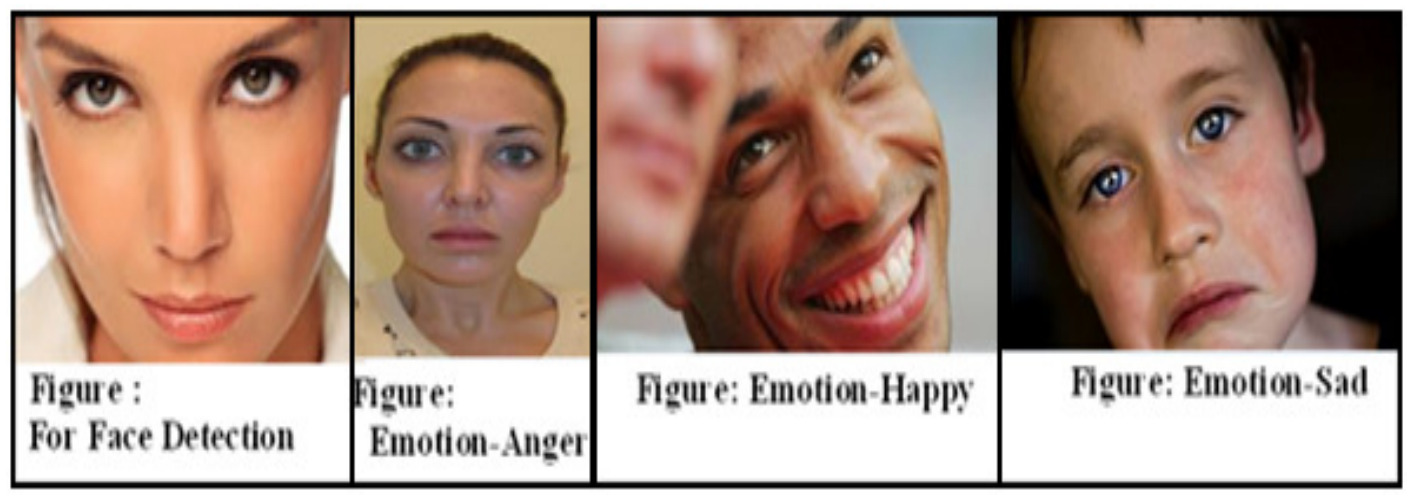


Table 5.2: Input physical appearance images

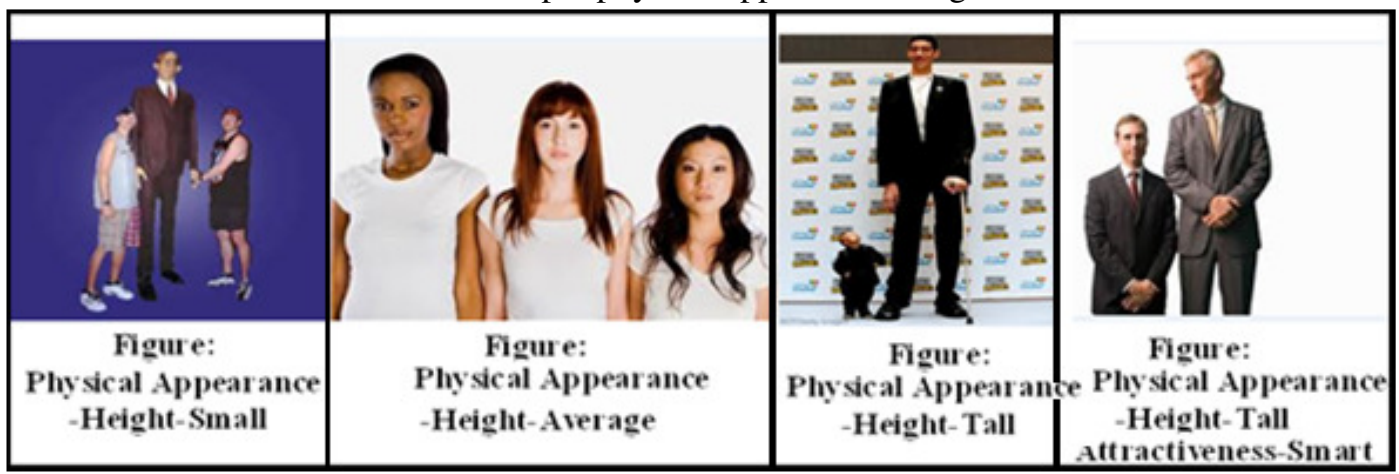

Table 5.3: Input body shape images

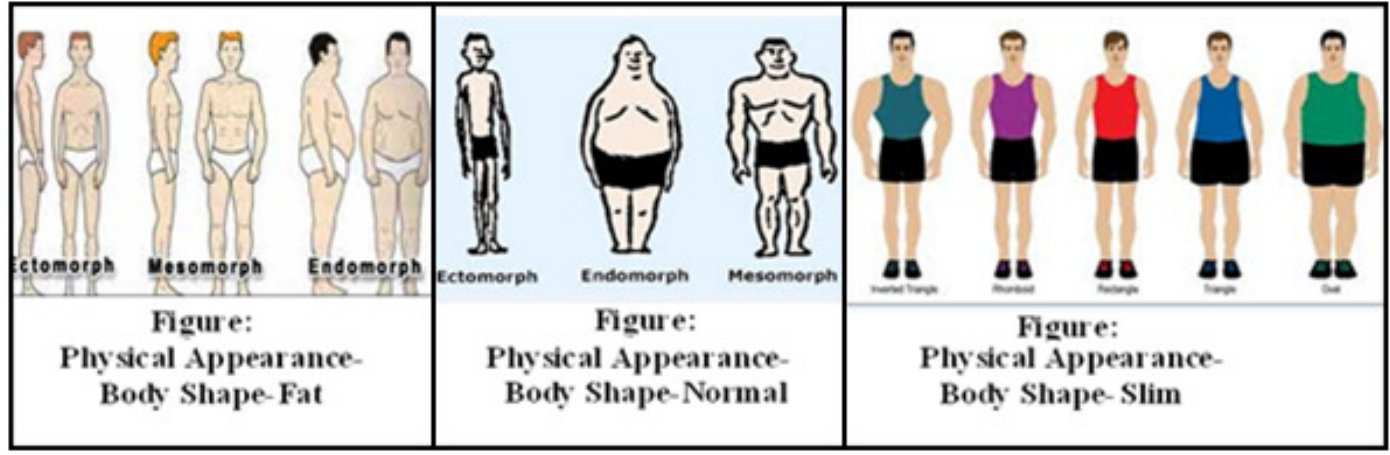

The simulated results of our proposed method are shown in the figure 5.4:

(a) Face Detection: Like: Eyes, Mouth, Lips, Ears and Nose.

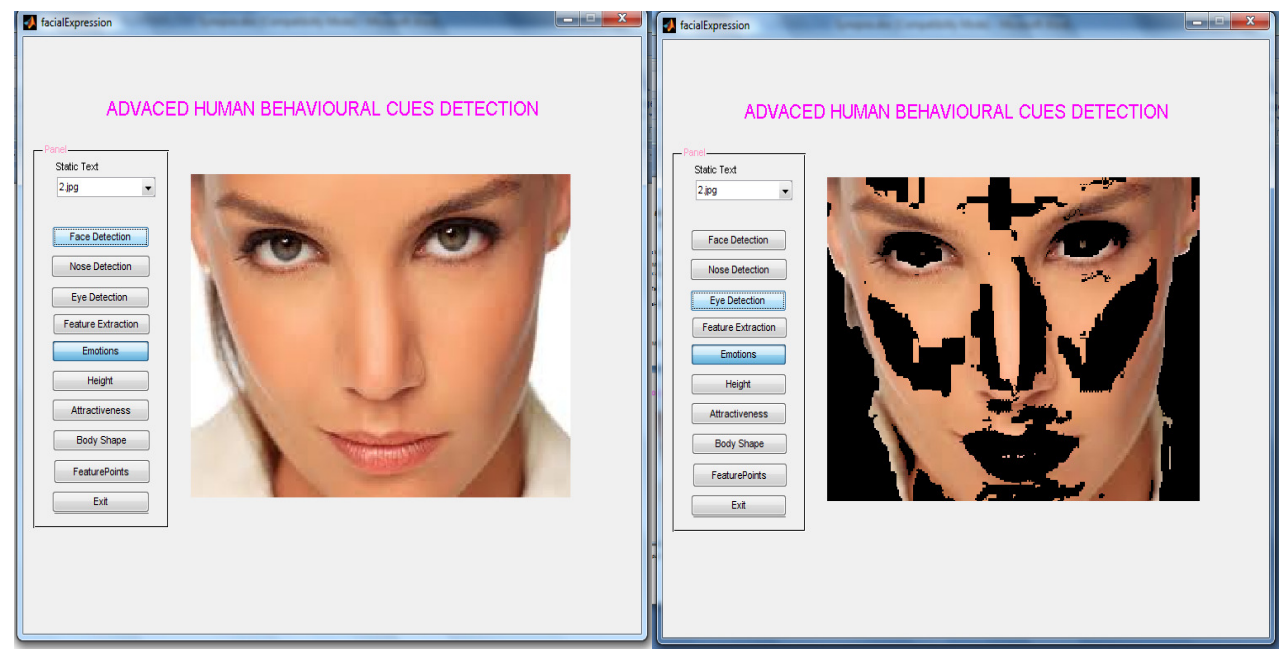

Figure 5.4(a): Input Image with Face

Figure 5.4(b): Eyes, Mouth based on Nose location,

Detection based on Nose Detection 
(b) Emotional Assessments: Like: Anger, Happy, Sadness and Neutral.

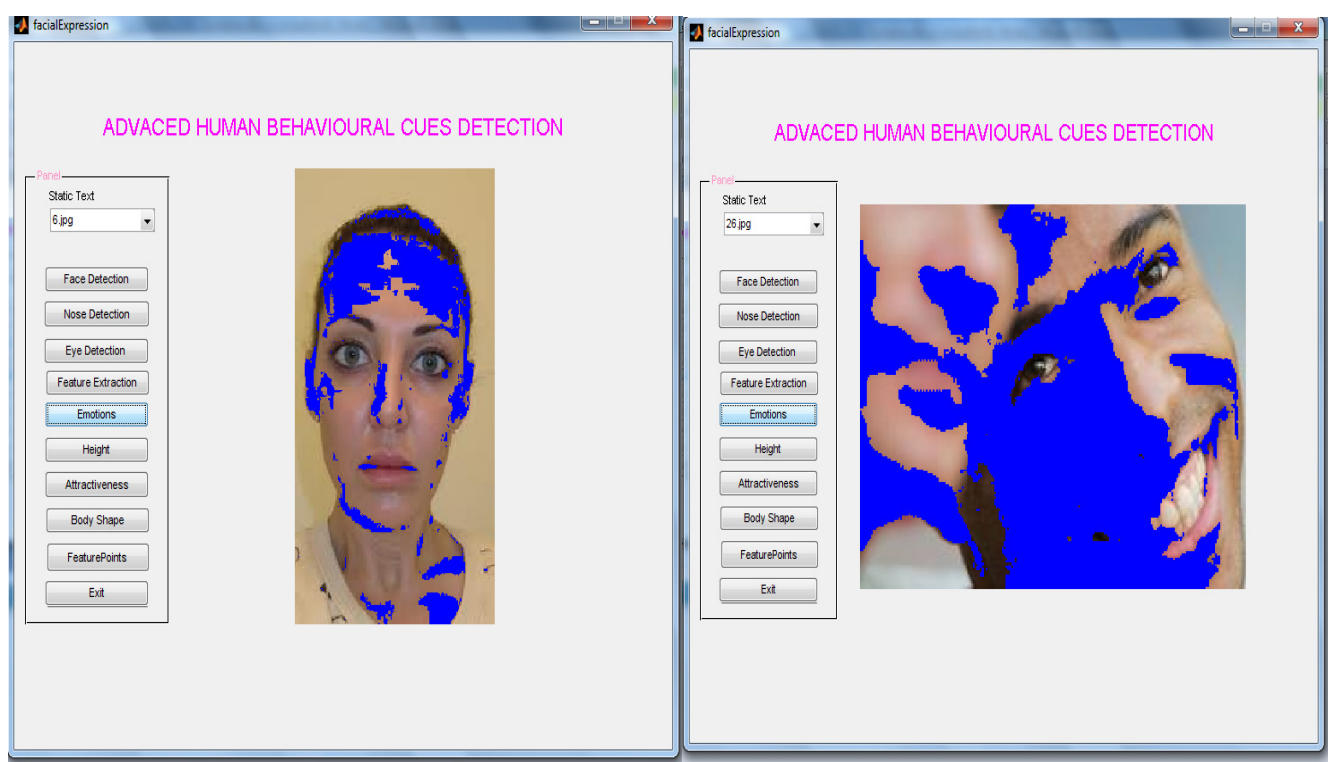

Figure 5.4(c): Emotion-Anger

Figure 5.4(d): Emotions- Happy

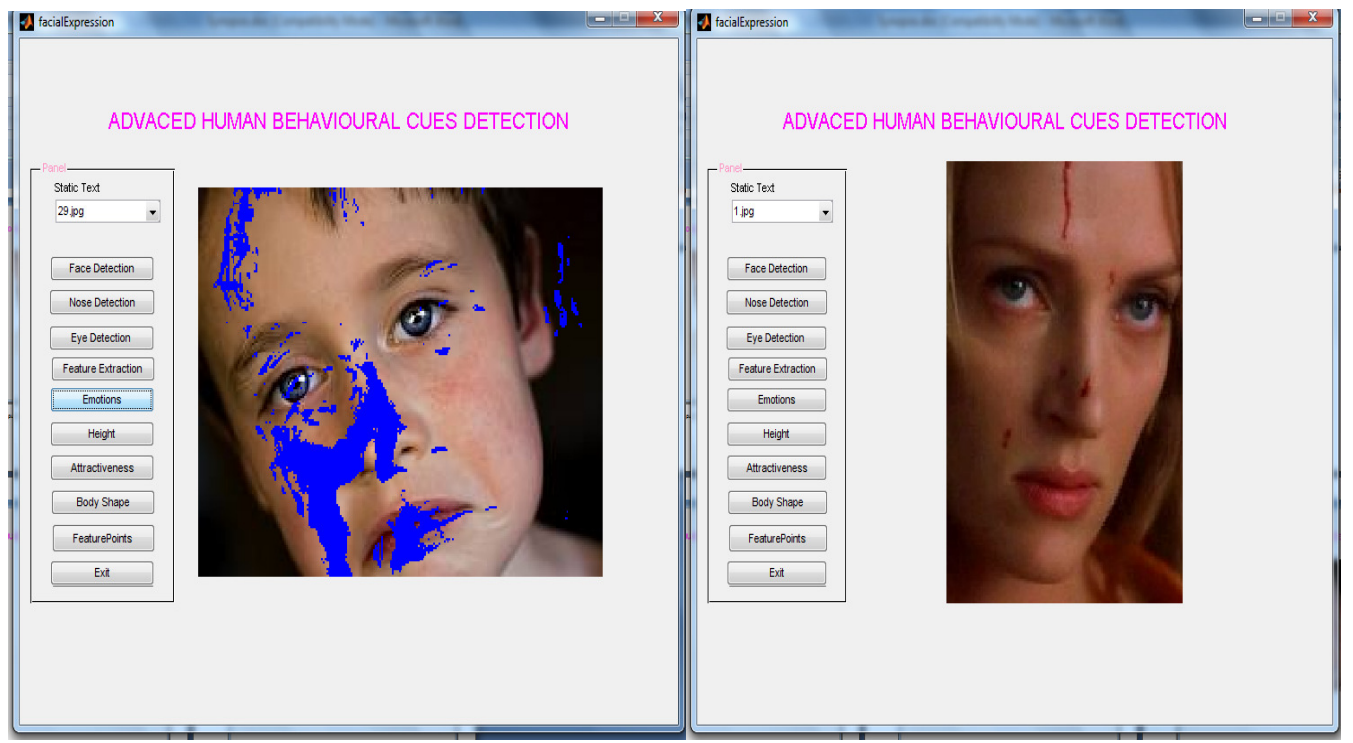

Figure 5.4(e): Emotions-SadFigure

Figure 5.4(f):Emotions-Neutral

(c) Physical Appearance :

1. Height: Small-Average-Tall.

2. Attractiveness: Smart

3. Body shape: Slim-Normal-Flat 
International Journal on Soft Computing, Artificial Intelligence and Applications (IJSCAI), Vol.2, No.1, Feb 2013

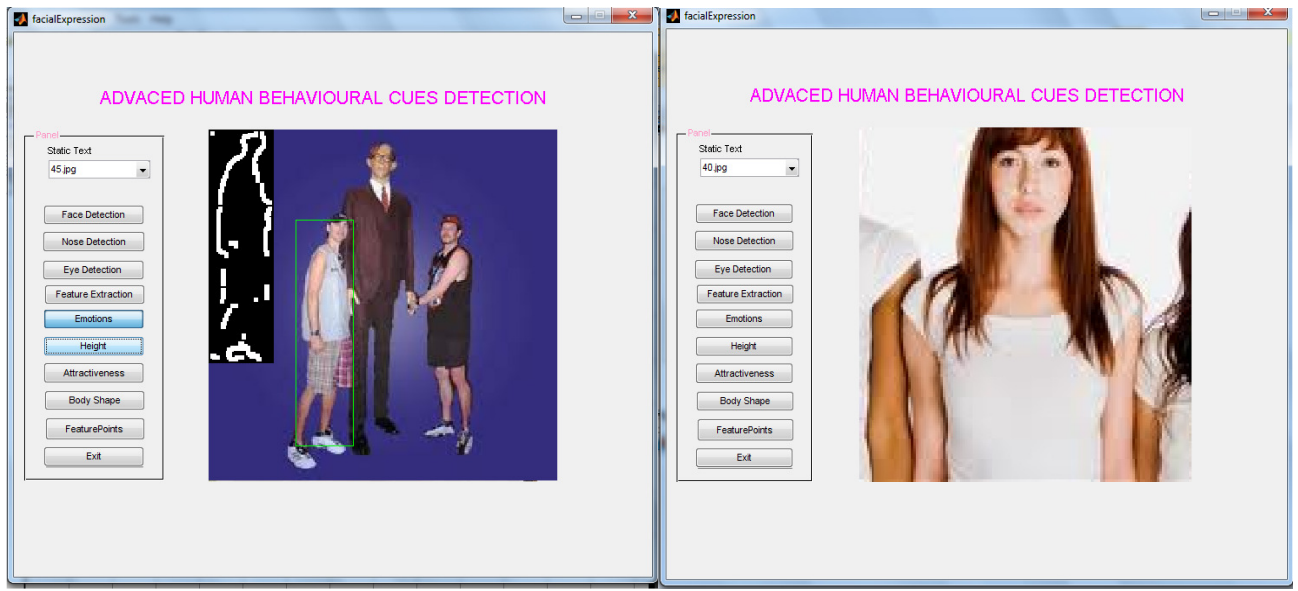

Figure 5.4(g): Physical Appearance-Height-Small Figure 5.4(h): Physical Appearance-Height-Average

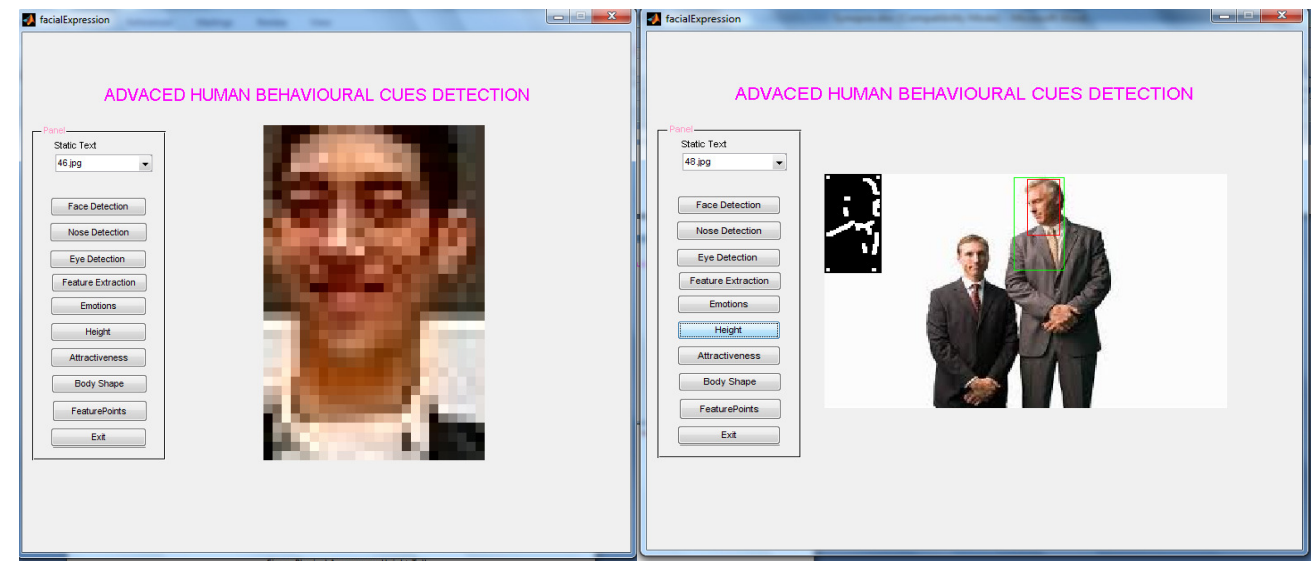

Figure 5.4(i): Physical Appearance-Height-Tall Figure5.4(j): Physical Appearance- Height-Tall and Attractiveness-Smart

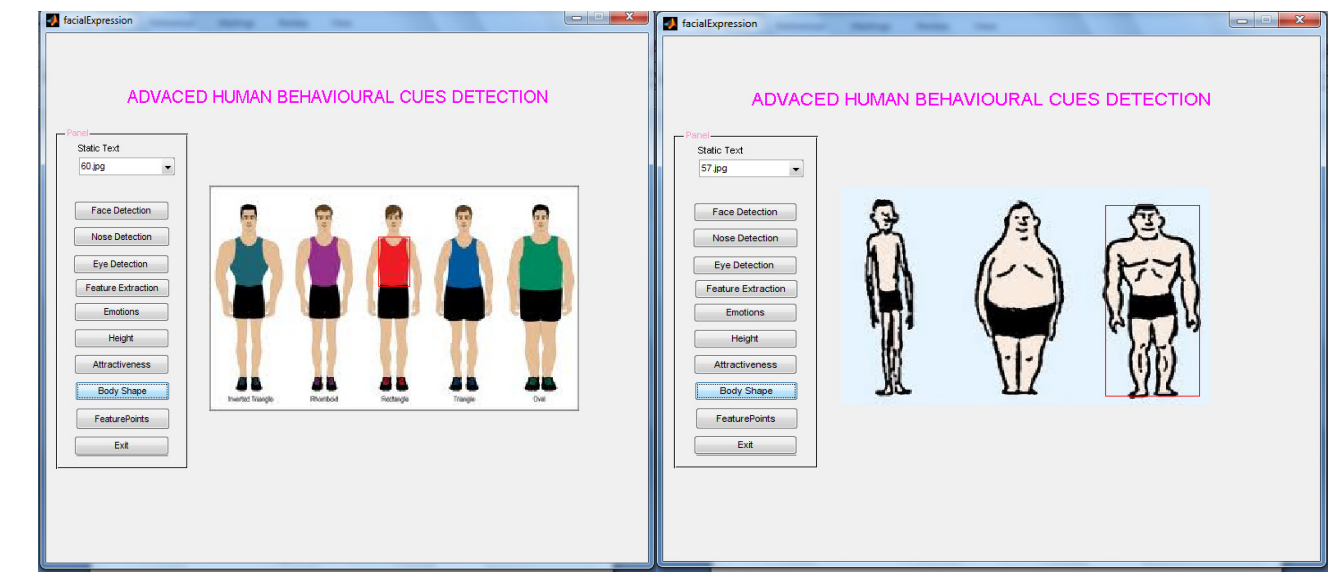

Figure 5.4(k):Physical Appearance-Body Shape-Slim Figure 5.4(1): Physical Appearance-Body Shape-Normal 


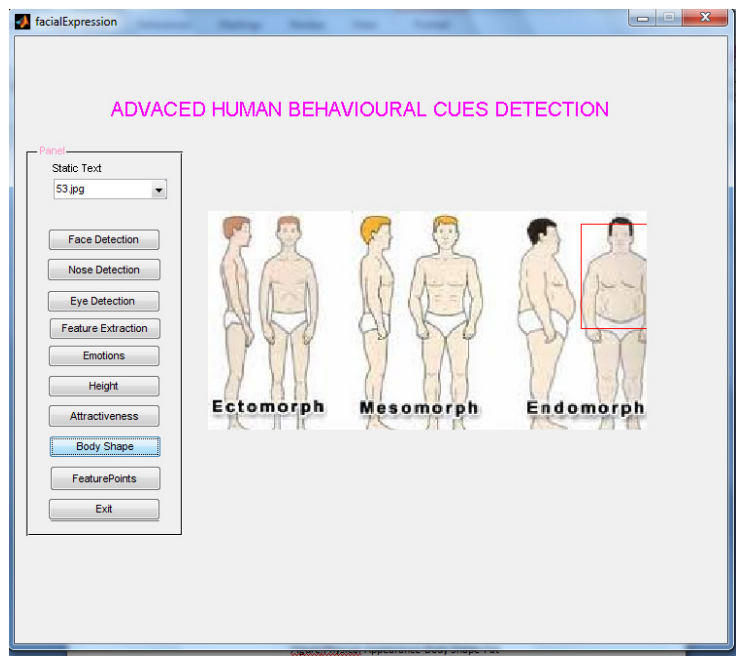

Figure 5.4(m): Physical Appearance-Body Shape-Fat

Above simulated results clearly shows the fastness and accuracy in extracting the social signal features like face, emotion and personality of the given single or group image. Our proposed shown good results in detecting the percentage of emotions between Anger, Happy, Sadness and Neutral. These results are very accurate compared with the other techniques.

\section{DISCUSSIONS}

The proposed method of human behavior cues detection has shown a simplest way to analyze the features of social signal processing using mat lab guisimulink. Few feature points are detected in all the images, which classifies the image with its feature.

\section{CONCLUSIONS}

Our method of human cues detection involving face detection, emotional assessment and physical appearances had shown a path for research work in the area of social signal processing. The processing time of the feature extraction and physical appearance is very less, making the system to become fast comparatively. The multiple facial and appearance images are analyzed individually making the method so flexible with the application point of view. Our proposed method can be applicable in detecting the emotions of different people in surveillance areas.

\section{FUTURE RESEARCH WORK}

Flexibility in our proposed method to detect the face and appearance of individual or group of people made to detect the motion of people in remote area where the behavior of the person can be analyzed. Our proposed method can be further extended to detect the hand gestures, walking and distance measurement of the given social images.

\section{REFERENCES}

[1]Alessandro Vinciarelli, MajaPantic and HervéBourlard, "Social signal processing: Survey of an emerging domain”, Image and Vision Computing 27 (2009) pp : 1743-1759.

[2]Georg Layher, HendrikLiebau, Robert Niese, Ayoub Al-Hamadi, Bernd Michaelis, and Heiko Neumann, "Robust Stereoscopic Head Pose Estimation in Human-Computer Interaction and a 
Unified Evaluation Framework"ICIAP 2011, Part I, LNCS 6978, pp. 227-236, 2011, SpringerVerlag Berlin Heidelberg 2011.

[3]M. Cristani, V. Murino, A. Vinciarelli, "Socially Intelligent Surveillance and Monitoring: Analysing Social Dimensions of Physical Space", 978-1-4244-7028-0/10 @2010 IEEE.

[4]Alessandro Vinciarelli, MajaPantic, Dirk Heylen, Catherine Pelachaud, Isabella Poggi, Francesca D'Errico and Marc Schr"oder, "Bridging the Gap Between Social Animal and Unsocial Machine: A Survey of Social Signal Processing", IEEE TRANSACTIONS ON AFFECTIVE COMPUTING, pp:1 -20.

[5]MajaPantic, Roderick Cowie, Francesca D'Errico, Dirk Heylen, Marc Mehu,CatherinePelachaud, Isabella Poggi, Marc Schroeder, and Alessandro Vinciarelli, "Social Signal Processing: The Research Agenda", a research paper.

[6]MajaPantic, "Machine Analysis of Facial Behaviour: Naturalistic \& DynamicBehaviour", a research paper.

\section{Authors.}

K.Sunilkumar received M.Tech degree in the area of VLSI DESIGN. Presently working as Asst. Professor in Department of Electronics \& Communication Engineering, Vinuthna Institute of Technology and science, Warangal.

Email: sunil_veena10@yahoo.co.in

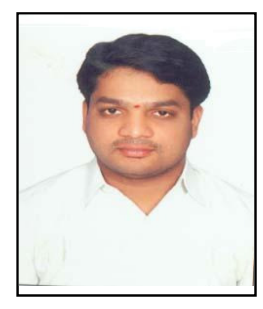

Dr. T. Kishore Kumar received Ph.D. degree in the area of signal processing. Presently working as Associate Professor in Department of Electronics \& Communication Engineering, National Institute of Technology (NIT), Warangal. He is faculty In-charge for Centre for Automation \& Instrumentation, NITW. He has 17 years of experience in teaching. His areas of interest are signal processing and speech processing. At present he is guiding $4 \mathrm{PhD}$ Scholars and 4 M.Tech Students. He has published 7 international Journals and 12 international conference papers during last five years. He attended different international conferences held at Czech

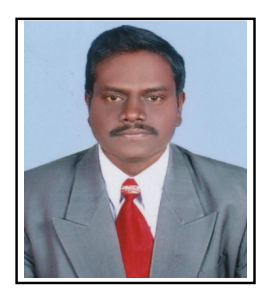
Republic, Israel \&Singapore. He has been deputed to ESIGELEC University, Rouen, France for taking assignment in Real Time Signal Processing. He has completed one in House R \& D Project in Speech Processing. He has delivered expert lectures for RCI (DRDO) \& ECIL Engineers. He is the Member of IEEE and also member of IETE. He is a Reviewer for TMH book on Signals and Systems. He is Member Board of Studies for various institutes.

Email: kishorefr@gmail.com 